\title{
TIGHT GALOIS CONNECTIONS AND COMPLETE DISTRIBUTIVITY
}

\author{
BY
}

\section{GEORGE N. RANEY}

1. Introduction. This paper describes a certain class of Galois connections and discusses its relations with the property of complete distributivity in complete lattices. A procedure for constructing Galois connections between complete lattices is presented. The Galois connections constructed by this procedure are called tight Galois connections, and are characterized as those which satisfy certain identities. All closure operations on complete lattices are obtainable from tight Galois connections. If either of the complete lattices involved in a Galois connection is completely distributive, then the Galois connection is tight. Consequently, all Galois connections constructed by the known procedure of Birkhoff are tight.

The identity mapping from a complete lattice to its dual lattice always determines a Galois connection; this Galois connection is tight if and only if the complete lattice is completely distributive. This last observation leads to a characterization of completely distributive complete lattices solely in terms of the partial ordering on them. It also provides new insight into the structure of these lattices, and enables us to prove a representation theorem which is considerably more economical than the one previously known.

2. Definitions and notations. If $F$ is a family of subsets of a set, the intersection of $F$ is denoted by $\prod F$, and the union of $F$ is denoted by $\sum F$.

If $L$ is a complete lattice, then every subset $K$ of $L$ has a meet, which is denoted by $\cap K$, and a join, which is denoted by $U K$.

If $L$ is any partially ordered set, a subset $J$ of $L$ is called a lower semi-ideal of $L$ if $x \in J, y \in L$, and $y \leqq x$ together imply $y \in J$. The definition of upper semi-ideal is obtained by changing the direction of the inequality.

If $L$ and $M$ are complete lattices and $r: L \rightarrow M$ and $n: M \rightarrow L$ are mappings such that (1) $x_{1} \leqq x_{2}$ in $L$ implies $r\left(x_{1}\right) \geqq r\left(x_{2}\right)$ in $M$, (2) $y_{1} \leqq y_{2}$ in $M$ implies $n\left(y_{1}\right) \geqq n\left(y_{2}\right)$ in $L$, (3) $n r(x) \geqq x$ in $L$, and (4) $r n(y) \geqq y$ in $M$, then the pair $[r, n]$ is called a Galois connection between $L$ and $M$. The theory of Galois connections has been developed by Ore, Birkhoff, and Everett $[1 ; 2 ; 3]$. Recently it has been observed $[4 ; 5]$ that Galois connections between $L$ and $M$ correspond in a one-to-one manner with the complete join homomorphisms from $L$ into the dual of $M$. In particular, if $A \subset L$, then $r(\cup A)=\bigcap\{r(a) \mid a \in A\}$.

If $L$ and $M$ are complete lattices, then $L \times M$ is the complete lattice whose elements are the pairs $(l, m)$ with $l \in L$ and $m \in M$, where $\left(l_{1}, m_{1}\right) \leqq\left(l_{2}, m_{2}\right)$ means that $l_{1} \leqq l_{2}$ and $m_{1} \leqq m_{2}$.

Received by the editors May 2, 1960. 
3. Tight Galois connections. Let $L$ and $M$ be complete lattices. Let $\theta$ be any subset of $L \times M$. Define $\theta^{+}$to be the set of $(l, m)$ in $L \times M$ such that for every $(u, v) \in \theta$ either $l \leqq u$ or $m \leqq v$. Define $\theta^{*}$ to be the set of $(l, m)$ in $L \times M$ such that for every $(u, v) \in \theta$ either $u \leqq l$ or $v \leqq m$. Then the following statements are readily seen to hold for any subsets $\theta, \theta_{1}, \theta_{2}$ of $L \times M$ : (1) $\theta^{+}$is a lower semi-ideal in $L \times M$, (2) $\theta^{*}$ is an upper semi-ideal in $L \times M$, (3) if $\theta_{1} \subset \theta_{2}$, then $\theta_{2}^{+} \subset \theta_{1}^{+}$and $\theta_{2}^{*} \subset \theta_{1}^{*}$, (4) $\theta \subset \theta^{*+}$ and $\theta \subset \theta^{+*}$. It follows that if $C$ is the complete lattice whose elements are the subsets of $L \times M$ partially ordered by set inclusion, then $+: C \rightarrow C$ and ${ }^{*}: C \rightarrow C$ together form a Galois connection. From the theory of Galois connections it is known that for any $\theta \subset L \times M$ both $\theta^{+}=\theta^{+*+}$ and $\theta^{*}=\theta^{*+*}$, that $+^{*}$ and ${ }^{*}+$ are closure operations on $C$ with closed sets of the forms $\theta^{*}$ and $\theta^{+}$respectively, and that there is a dual isomorphism between the complete lattices of closed sets under these two closure operations.

Birkhoff $[2$, p. 54] has described a construction under which each binary relation $\rho$ between. two sets $A$ and $B$ induces a Galois connection between the complete lattice of all subsets of $A$ and the complete lattice of all subsets of $B$. The Galois connection $\left[+,{ }^{*}\right]$ is induced in this manner by the binary relation $\beta$ between $L \times M$ and $L \times M$, if $\left(l_{1}, m_{1}\right) \beta\left(l_{2}, m_{2}\right)$ is taken to mean that either $l_{1} \leqq l_{2}$ or $m_{1} \leqq m_{2}$.

THEOREM 1. Let $L$ and $M$ be complete lattices and let $\theta \subset L \times M$. For $x \in L$ define $r(x)=\bigcup\left\{y \mid(x, y) \in \theta^{+}\right\}$and for $y \in M$ define $n(y)=\bigcup\left\{x \mid(x, y) \in \theta^{+}\right\}$. Then $[r, n]$ is a Galois connection between $L$ and $M$, and $\theta^{+}$consists of the pairs $(l, m)$ such that $(l, m) \leqq(u, v)$ for some pair $(u, v)$ such that $v=r(u)$ and $u=n(v)$.

Proof. If $x_{1} \leqq x_{2}$ in $L$, then since $\theta^{+}$is a lower semi-ideal in $L \times M, r\left(x_{2}\right)$ $=U\left\{y \mid\left(x_{2}, y\right) \in \theta^{+}\right\} \leqq U\left\{y \mid\left(x_{1}, y\right) \in \theta^{+}\right\}=r\left(x_{1}\right)$ in $M$. Similarly, if $y_{1} \leqq y_{2}$ in $M$, then $n\left(y_{2}\right) \leqq n\left(y_{1}\right)$ in $L$. If $x \in L$ and $(x, r(x))$ is not in $\theta^{+}$, then there is a $(u, v) \in \theta$ such that $x$ not $\leqq u$ and $r(x)$ not $\leqq v$. By the definition of $r(x)$, this would imply that there is a $y$ such that $(x, y) \in \theta^{+}$and $x$ not $\leqq u$ and $y$ not $\leqq v$. This would contradict the definition of $\theta^{+}$, and we conclude that $(x, r(x))$ $\in \theta^{+}$. Therefore $n r(x)=\bigcup\left\{t \mid(t, r(x)) \in \theta^{+}\right\} \geqq x$. Similarly, if $y \in M$, then $r n(y) \geqq y$, and it is proved that $[r, n]$ is a Galois connection between $L$ and $M$. If $(l, m) \in \theta^{+}$, then $(l, m) \leqq(l, r(l)) \leqq(n r(l), r(l))=(n r(l), r n r(l))$. If $(l, m)$ $\leqq(u, v)$ where $v=r(u)$, then since $(u, v)=(u, r(u)) \in \theta^{+}$and $\theta^{+}$is a lower semiideal, $(l, m) \in \theta^{+}$.

Theorem 1 shows that every relation $\theta$ between two complete lattices $L$ and $M$ determines, using $\theta^{+}$, a Galois connection $[r, n]$ between $L$ and $M$. Dually, every relation $\theta$ between $L$ and $M$ determines, using $\theta^{*}$, a Galois connection between the dual of $L$ and the dual of $M$.

The construction described in Theorem 1 differs from that of Birkhoff. In Birkhoff's construction a relation between two sets $A$ and $B$ induces a 
Galois connection between the family of all subsets of $A$ and the family of all subsets of $B$. In the present construction a relation between two complete lattices $L$ and $M$ induces a Galois connection between the same two lattices, and it is not required that either of the lattices be the lattice of all subsets of a set.

C. J. Everett [3, p. 517] has proved that if $A$ and $B$ are sets, then every Galois connection between the family of all subsets of $A$ and the family of all subsets of $B$ can be obtained using Birkhoff's construction. One wonders whether, for arbitrary complete lattices $L$ and $M$, every Galois connection between $L$ and $M$ can be obtained by the present construction. Without some further condition the answer is in the negative; the identity mapping of the five element nonmodular lattice onto its dual provides one counterexample. It will be shown, however, that whenever at least one of the lattices $L$ and $M$ is completely distributive, every Galois connection between $L$ and $M$ is obtainable by the present construction. Since the lattice of all subsets of a set is completely distributive, every Galois connection obtainable using Birkhoff's construction is also obtainable using the present construction. It will also become apparent that there are certain identities which are satisfied by all Galois connections produced by either Birkhoff's construction or the present construction but are not satisfied by all Galois connections.

THEOREM 2. If $[r, n]$ is a Galois connection between complete lattices $L$ and $M$, then the following four conditions are equivalent:

(I) Therc exists a subset $\theta$ of $L \times M$ such that for every $x \in L, r(x)$ $=U\left\{y \mid(x, y) \in \theta^{+}\right\}$.

(II) For every $x \in L, r(x)=\bigcap\{\cup\{r(t) \mid t$ not $\leqq u\} \mid u$ not $\geqq x\}$.

(III) There exists a subset $\theta$ of $L \times M$ such that for every $y \in M, n(y)$ $=U\left\{x \mid(x, y) \in \theta^{+}\right\}$.

(IV) For every $y \in M, n(y)=\bigcap\{\cup\{n(t) \mid t$ not $\leqq v\} \mid v$ not $\geqq y\}$.

If either $L$ or $M$ is completely distributive, then all four conditions are satisfied.

Proof. (I) implies (II). For every $x \in L, r(x)=U\left\{y \mid(x, y) \in \theta^{+}\right\}$ $=\bigcup\left\{y \mid(x, y) \in \theta^{+*+}\right\}=\bigcup\left\{y \mid\right.$ for every $(u, v) \in \theta^{+*}$, if $x$ not $\leqq u$, then $\left.y \leqq v\right\}$ $=\bigcap\left\{v \mid\right.$ for some $u$ not $\left.\geqq x,(u, v) \in \theta^{+*}\right\}=\bigcap\left\{\cap\left\{v \mid(u, v) \in \theta^{+*}\right\} \mid u\right.$ not $\left.\geqq x\right\}$ $=\bigcap\{\cup\{r(t) \mid t$ not $\leqq u\} \mid u$ not $\geqq x\}$.

(II) implies (I). Let $\theta=\{(u, v) \mid v \geqq U\{r(t) \mid t$ not $\leqq u\}\}$. Then $\cup\left\{y \mid(x, y) \in \theta^{+}\right\}=\bigcup\{y \mid$ whenever $v \geqq \bigcup\{r(t) \mid t$ not $\leqq u\}$ and $x$ not $\leqq u$, then $y \leqq v\}=\bigcap\{\bigcup\{r(t) \mid t$ not $\leqq u\} \mid u$ not $\geqq x\}=r(x)$.

In a similar manner, it can be proved that (III) and (IV) are equivalent.

For any Galois connection $[r, n], n(y)=\bigcup\{x \mid r(x) \geqq y\}$ and $r(x)$ $=\bigcup\{y \mid n(y) \geqq x\}$. If $r(x)=\bigcup\left\{y \mid(x, y) \in \theta^{+}\right\}$, then $(x, y) \in \theta^{+}$if and only if $y \leqq r(x)$. Therefore $n(y)=\bigcup\left\{x \mid(x, y) \in \theta^{+}\right\}$. This shows that (I) implies (III). Similarly it can be shown that (III) implies (I).

The inequality $r(x) \leqq \bigcap\{U\{r(t) \mid t$ not $\leqq u\} \mid u$ not $\geqq x\}$ holds for any 
complete lattices $L$ and $M$. It will be shown next that if $M$ is completely distributive, then the reverse inequality also holds.

First let us show that if $L$ is any complete lattice and $x \in L$; and if $f$ is a function which assigns to each $t$ not $\geqq x$ an element $f(t)$ such that $f(t)$ not $\leqq t$, then $U\{f(t) \mid t$ not $\geqq x\} \geqq x$. For let $t_{0}=U\{f(t) \mid t$ not $\geqq x\}$; if $t_{0}$ not $\geqq x$, then according to the hypothesis on $f, f\left(t_{0}\right)$ not $\leqq t_{0}$; but according to the definition of $t_{0}, f\left(t_{0}\right) \leqq t_{0}$, and we have a contradiction.

Now suppose that $M$ is completely distributive and let $F$ be the set of functions $f$ such that $f$ assigns to each $u$ not $\geqq x$ an element $f(u)$ such that $f(u)$ not $\leqq u$. Then $\bigcap\{\cup\{r(t) \mid t$ not $\leqq u\} \mid u$ not $\geqq x\}=U\{\cap\{r(f(u)) \mid u$ not $\geqq x\} \mid f \in F\}=\bigcup\{r(\cup\{f(u) \mid u$ not $\geqq x\}) \mid f \in F\} \leqq r(x)$, by the result of the preceding paragraph. Therefore, complete distributivity of $M$ implies condition (II).

In a similar manner it can be proved that complete distributivity of $L$ implies condition (IV).

A Galois condition satisfying the conditions (I)-(IV) of Theorem 2 will be called a tight Galois connection.

One reason for studying Galois connections is that they lead to closure operations. The following theorem shows that we will obtain all closure operations even if we restrict our attention to tight Galois connections.

Theorem 3. Let $L$ be a complete lattice and let $C$ be the set of closed elements of $L$ under some closure operation on $L$. Let $M$ be the complete lattice of all subsets of $C$. For $x \in L$, define $r(x)$ to be the set of closed elements $c$ of $L$ such that $x \leqq c$. If $y \in M$, define $n(y)$ to be the meet of $y$ in L. Then $[r, n]$ is a tight Galois connection between $L$ and $M$, and an element $x$ of $L$ is a member of $C$ if and only if $x=n r(x)$.

Proof. If $x_{1} \leqq x_{2}$ in $L$ and $c \in C$, then $x_{2} \leqq c$ implies $x_{1} \leqq c$. Therefore $r\left(x_{2}\right)$ $\leqq r\left(x_{1}\right)$. If $y_{1} \leqq y_{2}$ in $M$, then $n\left(y_{2}\right)=\bigcap y_{2} \leqq \bigcap y_{1}=n\left(y_{1}\right)$. If $x \in L$, then $n r(x)$ is the meet of the set of closed elements $c$ such that $x \leqq c$. Therefore $x \leqq n r(x)$. Furthermore $n r(x)$ is the closure of $x$ and the closed elements $x$ of $L$ are precisely those for which $x=n r(x)$. If $y \in M$, then $r n(y)$ is the set of closed elements $c$ such that $\cap y \leqq c$. Therefore $y \leqq r n(y)$. This shows that $[r, n]$ is a Galois connection. Since $M$ is the lattice of all subsets of a set, $M$ is completely distributive, and $[r, n]$ is a tight Galois connection.

From Theorem 2 it follows that if a complete lattice is completely distributive, then any Galois connection in which it is involved is a tight Galois connection. The following theorem shows that for any complete lattice which is not completely distributive there is at least one Galois connection involving it which is not tight.

THEOREM 4. Let $L$ be a complete lattice and let $L^{D}$ be its dual lattice. Let $I: L \rightarrow L^{D}$ be defined by $I(x)=x$ for $x \in L$. Let $I^{D}: L^{D} \rightarrow L$ be defined by $I^{D}(y)$ 
$=y$ for $y \in L^{D}$. Then $\left[I, I^{D}\right]$ is a Galois connection between $L$ and $L^{D}$ which is tight if and only if $L$ is completely distributive.

Proof. That $\left[I, I^{D}\right]$ is a Galois connection is easily checked. If $L$ is completely distributive, then $\left[I, I^{D}\right]$ is tight by Theorem 2 . It only remains to be proved that if $\left[I, I^{D}\right]$ is tight, then $L$ is completely distributive. For the sake of clarity, all inequalities will be expressed in terms of the partial ordering on $L$; no further reference to the ordering on $L^{D}$ will be made.

For $x \in L$, define $K(x)$ to be the intersection of the family of lower semiideals $J$ in $L$ such that $U J \geqq x$. It is known [6] that $L$ is completely distributive if and only if $U K(x)=x$ for every $x \in L$. Make the further definitions: $R(a)=\bigcup\{t \mid t$ not $\geqq a\}, N(a)=\bigcap\{t \mid t$ not $\leqq a\}$, for $a \in L$.

First it will be proved that $\bigcup\{N(a) \mid a$ not $\geqq x\}=\bigcup\{b \mid R(b)$ not $\geqq x\}$, for every $x \in L$. Let $b$ be such that $R(b)$ not $\geqq x$. Then there is an $a$, namely $R(b)$, such that $a$ not $\geqq x$ and $b \leqq N(a)$. Hence $U\{N(a) \mid a$ not $\geqq x\}$ $\geqq \cup\{b \mid R(b)$ not $\geqq x\}$. Next let $a$ be such that $a$ not $\geqq x$. Then $R N(a)$ not $\geqq x$, and there is a $b$, namely $N(a)$, such that $b \geqq N(a)$ and $R(b)$ not $\geqq x$. Hence $U\{N(a) \mid a$ not $\geqq x\} \leqq U\{b \mid R(b)$ not $\geqq x\}$.

Second it will be proved that for every $x \in L, w \in K(x)$ if and only if $R(w)$ not $\geqq x$. If $x \leqq R(w)$, then $x \leqq U\{t \mid t$ not $\geqq w\}$. Since $\{t \mid t$ not $\geqq w\}$ is a lower semi-ideal, and $w$ is not a member of it, $w$ cannot be a member of $K(x)$. Conversely, if $w$ is not a member of $K(x)$, then there is a lower semi-ideal $J$ such that $U J \geqq x$ and $w$ is not in $J$. Then $J \subset\{t \mid t$ not $\geqq w\}$, and $U J$ $\leqq \bigcup\{t \mid t$ not $\geqq w\}=R(w)$. Therefore, $x \leqq R(w)$.

The hypothesis that $\left[I, I^{D}\right]$ is tight can be expressed in the form $x=\bigcup\{\cap\{t \mid t$ not $\leqq u\} \mid u$ not $\geqq x\}$, by condition (II) of Theorem 2. Using the results of the two preceding paragraphs, we then obtain $x=\bigcup\{N(u) \mid u$ not $\geqq x\}=\bigcup\{w \mid R(w)$ not $\geqq x\}=\bigcup(K(x))$, for every $x \in L$. It follows that $L$ is completely distributive.

The following theorem characterizes completely distributive complete lattices solely in terms of the partial ordering relation.

THEOREM 5. In order that the complete lattice $L$ be completely distributive it is necessary and sufficient that whenever $u$ not $\leqq v$ in $L$ there exist elements $p$ and $q$ in $L$ such that $u$ not $\leqq p$ and $v$ not $\geqq q$ and for every $x \in L$ either $x \leqq p$ or $x \geqq q$.

Proof. By Theorem 4, $L$ is completely distributive if and only if $\left[I, I^{D}\right]$ is tight. By definition, $\left[I, I^{D}\right]$ is tight if and only if there exists a relation $\theta \subset L \times L^{D}$ such that $x=I(x)=\bigcap\left\{y \mid(x, y) \in \theta^{+}\right\}$, and $y=I^{D}(y)$ $=\bigcup\left\{x \mid(x, y) \in \theta^{+}\right\}$. Here, as before, our statements are expressed in terms of the ordering on $L$. By Theorem $1, \theta^{+}$consists of the pairs $(u, v)$ such that $u \leqq x$ and $v \geqq x$ for some $x \in L$. Simplifying, $\theta^{+}$consists of the pairs $(u, v)$ such that $u \leqq v$. If $u$ not $\leqq v$, then $(u, v)$ is not in $\theta^{+}$, and since $\theta^{+}=\theta^{+*+},(u, v)$ is not in $\theta^{+*+}$. This implies that there is a pair $(p, q)$ in $\theta^{+*}$ such that $u$ not 
$\leqq p$ and $v$ not $\geqq q$. But $(p, q)$ is in $\theta^{+*}$ if and only if whenever $g \leqq h$ then either $p \geqq g$ or $q \leqq h$. It then follows easily that $(p, q)$ is in $\theta^{+*}$ if and only if for every $x \in L$ either $x \leqq p$ or $x \geqq q$.

4. Representation theory. Let $L$ be any complete lattice. An ordered pair $(p, q)$ of elements of $L$ will be called a blanket if and only if for every $x \in L$, either $x \leqq p$ or $x \geqq q$.

A blanket $(p, q)$ will be called a minimax blanket if and only if $p_{1}<p$ implies that $\left(p_{1}, q\right)$ is not a blanket and $q_{1}>q$ implies that $\left(p, q_{1}\right)$ is not a blanket.

A blanket $(p, q)$ will be said to separate the elements $u$ and $v$ of $L$ if and only if $u$ not $\leqq p$ and $v$ not $\geqq q$.

THEOREM 6. Let L be a complete lattice. For $x \in L$, define $R(x)=\bigcup\{t \mid$ tnot $\geqq x\}$ and $N(x)=\bigcap\{t \mid t$ not $\leqq x\}$. Then $[R, N]$ is a tight Galois connection between $L$ and its dual. $A$ blanket $(p, q)$ is a minimax blanket if and only if $p=R(q)$ and $q=N(p)$, or, equivalently, $(p, q)=(R(x), N R(x))$ for some $x$. If there is a blanket which separates the elements $u$ and $v$ of $L$, then there is a minimax blanket which separates $u$ and $v$.

Proof. Using the notation of Theorem 1, let $\theta=\{(t, t) \mid t \in L\}$. Then $\theta^{+}=\{(x, y) \mid$ for every $t \in L$, either $x \leqq t$ or $y \geqq t\}$. Now $R(x)=U\{t \mid t$ not $\geqq x\}$ $=\bigcap\{y \mid$ if $t$ not $\geqq x$, then $y \geqq t\}=\bigcap\left\{y \mid(x, y) \in \theta^{+}\right\}$. Similarly, $N(y)$ $=U\left\{x \mid(x, y) \in \theta^{+}\right\}$. It follows from Theorems 1 and 2 that $[R, N]$ is a tight Galois connection between $L$ and its dual. Therefore $R=R N R$ and a pair $(p, q)$ satisfies $p=R(q)$ and $q=N(p)$ if and only if $(p, q)=(R(x), N R(x))$ for some $x$.

We next show that for every $x \in L,(R(x), N R(x))$ is a minimax blanket. By the definition of $N$, if $u$ not $\leqq R(x)$, then $u \geqq N R(x)$. Therefore $(R(x), N R(x))$ is a blanket. If $p<R(x)$, then by the definition of $R, p$ not $\geqq t$ for some $t$ not $\geqq x$. Since $N R(x) \geqq x, t$ not $\geqq N R(x)$ and $(p, N R(x))$ is not a blanket. If $q>N R(x)$, then $q$ not $\leqq t$ for some $t$ not $\leqq R(x)$ and $(R(x), q)$ is not a blanket. Therefore, $(R(x), N R(x))$ is a minimax blanket.

If $(p, q)$ is any blanket, then whenever $x$ not $\geqq q, x$ must be $\leqq p$. Therefore $p \geqq R(q)$. Similarly, $q \leqq N(p)$. Now $(R(q), q)$ and $(p, N(p))$ are blankets. Therefore, if $p>R(q)$ or $q<N(p)$, then $(p, q)$ is not a minimax blanket. It follows that if $(p, q)$ is a minimax blanket, then $p=R(q)$ and $q=N(p)$.

Let $u$ and $v$ be elements of $L$ which are separated by the blanket $(p, q)$. Since $p \geqq R(q)$ and $u$ not $\leqq p, u$ not $\leqq R(q)$; and since $N R(q) \geqq q$ and $v$ not $\geqq q, v$ not $\geqq N R(q)$. Therefore $u$ and $v$ are separated by the minimax blanket $(R(q), N R(q))$.

Completely distributive complete lattices can now be characterized by the following condition: whenever $u$ not $\leqq v$ in $L$, there is a minimax blanket $(R(x), N R(x))$ in $L$ which separates $u$ and $v$. In the representation theory for these lattices the minimax blankets are useful, since they are readily located 
in specific examples and since they have the separation property just expressed. Some time ago, in answer to a question of Birkhoff, a subdirectunion representation theorem for completely distributive complete lattices was proved [6]. Now, using minimax blankets, it is possible to render the proof of this theorem more perspicuous while greatly reducing the nunber of chains required in the representation.

THEOREM 7. Every completely distributive complete lattice is isomorphic with a closed sublattice of the direct union of a family of complete chains.

Proof. Let $L$ be a completely distributive complete lattice and let $P$ be the set of all minimax blankets $(R(t), N R(t))$ for $t \in L$. Define

$$
\left(R\left(t_{1}\right), N R\left(t_{1}\right)\right) \lambda\left(R\left(t_{2}\right), N R\left(t_{2}\right)\right)
$$

to mean that $R\left(t_{1}\right)$ not $\geqq N R\left(t_{2}\right)$. We show first that $\lambda$ is idempotent, $\lambda=\lambda \circ \lambda$.

If $\left(R\left(t_{1}\right), N R\left(t_{1}\right)\right) \lambda\left(R\left(t_{2}\right), N R\left(t_{2}\right)\right)$, then $N R\left(t_{2}\right)$ not $\leqq R\left(t_{1}\right)$. Since $L$ is completely distributive, there is a minimax blanket $(R(x), N R(x))$ which separates $N R\left(t_{2}\right)$ and $R\left(t_{1}\right)$. Then $N R\left(t_{2}\right)$ not $\leqq R(x)$ and $R\left(t_{1}\right)$ not $\geqq N R(x)$, so that $\left(R\left(t_{1}\right), N R\left(t_{1}\right)\right) \lambda(R(x), N R(x)) \lambda\left(R\left(t_{2}\right), N R\left(t_{2}\right)\right)$. Therefore, $\dot{\lambda} \subset \lambda \circ \lambda$.

Conversely, if $\left(R\left(t_{1}\right), N R\left(t_{1}\right)\right) \lambda(R(x), N R(x)) \lambda\left(R\left(t_{2}\right), N R\left(t_{2}\right)\right)$, then $R\left(t_{1}\right)$ not $\geqq N R(x)$, so that we must have $R(x) \geqq R\left(t_{1}\right)$. If $N R\left(t_{2}\right) \leqq R\left(t_{1}\right)$, then $N R\left(t_{2}\right) \leqq R(x)$. But $N R\left(t_{2}\right)$ not $\leqq R(x)$. Hence $N R\left(t_{2}\right)$ not $\leqq R\left(t_{1}\right)$, and $\left(R\left(t_{1}\right), N R\left(t_{1}\right)\right) \lambda\left(R\left(t_{2}\right), N R\left(t_{2}\right)\right)$. Therefore $\lambda \circ \lambda \subset \lambda$.

The relation $\lambda$ on the set $P$ is transitive and antisymmetric, but it is not necessarily a partial ordering, since it need not be reflexive. Nevertheless, we may define chains in $\lambda$ in the usual manner: a subset $C$ of $P$ is called a chain in $\lambda$ if and only if $B_{1} \in C$ and $B_{2} \in C$ implies either $B_{1}=B_{2}$ or $B_{1} \lambda B_{2}$ or $B_{2} \lambda B_{1}$. Furthermore, every chain in $\lambda$ is contained in a maximal chain in $\lambda$.

Let $\Gamma$ be the set of maximal chains in $\lambda$. If $C \in \Gamma$ and $a \in L$, let $m(C, a)$ be the set of $(R(t), N R(t)) \in C$ such that there exists an $(R(x), N R(x)) \in C$ such that $(R(t), N R(t)) \lambda(R(x), N R(x))$ and $R(x)$ not $\geqq a$. If $C \in \Gamma$, let $F_{C}=\{m(C, a) \mid a \in L\}$. We show next that $F_{C}$ is a complete chain in the relation of set inclusion on the set of subsets of $C$.

If $m(C, a)$ not $C m(C, b)$, then there exists an $(R(t), N R(t))$ which is a member of $m(C, a)$ but not a member of $m(C, b)$. If $(R(y), N R(y)) \in m(C, b)$, then we cannot have $(R(t), N R(t)) \lambda(R(y), N R(y))$; for, since $\lambda \circ \lambda \subset \lambda$, this would imply that $(R(t), N R(t)) \in m(C, b)$. Since we also cannot have $(R(t), N R(t)=(R(y), N R(y))$, we must have $(R(y), N R(y)) \lambda(R(t), N R(t))$ and $(R(y), N R(y)) \in m(C, a)$. It follows that $m(C, b) \subset m(C, a)$, and it has been proved that $F_{C}$ is a chain.

If $C \in \Gamma$ and $A \subset L$, then $\sum\{m(C, a) \mid a \in A\}=m(C, \cup A)$. For $(R(t), N R(t)) \lambda(R(x), N R(x))$ and $R(x)$ not $\geqq \cup A$ if and only if for some $a \in A$, $(R(t), N R(t)) \lambda(R(x), N R(x))$ and $R(x)$ not $\geqq a$. This proves that $F_{C}$ is a com- 
plete chain in which if $F \subset F_{C}, U F=\sum F$ and $\cap F=\sum\{m(C, b) \mid b \in L$ and $\left.m(C, b) \subset \prod F\right\}$.

Next it will be shown that if $C \in \Gamma$ and $A \subset L$ then $\cap\{m(C, a) \mid a \in A\}$ $=m(C, \cap A)$. If $(R(t), N R(t)) \in \cap\{m(C, a) \mid a \in A\}$, then there exists a $b \in L$ such that $(R(t), N R(t)) \in m(C, b)$ and $m(C, b) \subset \prod\{m(C, a) \mid a \in A\}$. Then $(R(t), \quad N R(t)) \in C$ and there exists an $(R(s), N R(s)) \in C$ such that $(R(t), N R(t)) \lambda(R(s), N R(s))$ and $R(s)$ not $\geqq b$. Since $\lambda \subset \lambda \circ \lambda$ there exist $(R(u), N R(u))$ and $(R(y), N R(y))$, both in $C$, such that $(R(t), N R(t))$ $\lambda(R(u), N R(u)) \lambda(R(y), N R(y)) \lambda(R(s), N R(s))$ and $R(s)$ not $\geqq b$. It follows that $(R(y), N R(y)) \in m(C, b)$; hence $(R(y), N R(y)) \in m(C, a)$ for every $a \in A$. This implies that for every $a \in A, R(y)$ not $\geqq a$, and we must have $N R(y) \leqq a$ for every $a \in A$. Therefore, $N R(y) \leqq \bigcap A$. Now $R(u)$ not $\geqq N R(y)$; hence $R(u)$ not $\geqq \cap A$. We conclude that $(R(t), N R(t)) \in m(C, \cap A)$. This proves that $\cap\{m(C, a) \mid a \in A\} \subset m(C, \cap A)$. Since $m(C, \cap A) \subset m(C, a)$ for every $a \in A$, we also have that $m(C, \cap A) \subset \cap\{m(C, a) \mid a \in A\}$.

Let $D$ be the direct union of the family of complete chains $\left\{F_{C} \mid C \in \Gamma\right\}$. For every $a \in L$, let $\phi_{a}$ be the member of $D$ such that for every $C \in \Gamma, \phi_{a}(C)$ $=m(C, a)$. Let $L^{*}=\left\{\phi_{a} \mid a \in L\right\}$. The mapping $a \rightarrow \phi_{a}$ is a one-to-one mapping of $L$ onto $L^{*}$. For suppose $a \neq b$, with $a$ not $\leqq b$. Then there is an $(R(t), N R(t))$ which separates $a$ and $b$, so that $a$ not $\leqq R(t)$ and $b$ not $\geqq N R(t)$. Now there is an $(R(l), N R(l))$ which separates $a$ and $R(t)$, and we have $a$ not $\leqq R(l)$ and $R(t)$ not $\geqq N R(l)$. Therefore $(R(t), N R(t)) \lambda(R(l), N R(l))$. Let $C_{1}$ be a maximal chain in $\lambda$ containing both $(R(t), N R(t))$ and $(R(l), N R(l))$. Then $(R(t), N R(t))$ is a member of $m\left(C_{1}, a\right)$. Since $b$ not $\geqq N R(t), b \leqq R(t)$, and it follows that $(R(t), N R(t))$ is not a member of $m\left(C_{1}, b\right)$. Therefore $m\left(C_{1}, a\right)$ $\neq m\left(C_{1}, b\right)$, and $\phi_{a} \neq \phi_{b}$.

Finally we prove that $L^{*}$ is a closed sublattice of $D$ and that the mapping $a \rightarrow \phi_{a}$ preserves joins and meets. If $A \subset L$ and $C \in \Gamma$, then $\cup\left\{\phi_{a} \mid a \in A\right\}(C)$ $=\bigcup\left\{\phi_{a}(C) \mid a \in A\right\}=\bigcup\{m(C, a) \mid a \in A\}=\sum\{m(C, a) \mid a \in A\}=m(C, \cup A)$ $=\phi_{\cup A}(C)$, and $\cap\left\{\phi_{a} \mid a \in A\right\}(C)=\bigcap\left\{\phi_{a}(C) \mid a \in A\right\}=\bigcap\{m(C, a) \mid a \in A\}$ $=m(C, \cap A)=\phi_{\cap A}(C)$. This completes the proof of Theorem 7 .

The present proof is based on the relation $\lambda$ between minimax blankets, while the proof given in [6] was based on the relation $\rho$ which holds between elements $x$ and $y$ of $L$ if and only if $x \in K(y)$, where $K(y)$ is defined as in the proof of Theorem 4. Making use of an observation made in that proof, we can describe the relation $\rho$ in terms of the function $R$, and we have that $x \rho y$ if and only if $R(x)$ not $\geqq y$. It can then be seen that each maximal chain in $\lambda$ determines a maximal chain in $\rho$ in the following manner: if $\{(R(t), N R(t)) \mid t \in T\}$ is a maximal chain in $\lambda$, indexed by $T$, then $\{N R(t) \mid t \in T\}$ is a maximal chain in $\rho$. Since different maximal chains in $\lambda$ determine different maximal chains in $\rho$, the number of maximal chains in $\lambda$ cannot exceed the number of maximal chains in $\rho$. Consideration of simple 
examples suggests that in many cases a substantial reduction in the number of chains will be achieved by using $\lambda$ instead of $\rho$. For example, if $L$ is the lattice of subsets of a set with $n$ elements, $n>1$, then there are only $n$ maximal chains in $\lambda$ while there are $n\left(2^{n-1}-1\right)$ maximal chains in $\rho$. Or if $L=U \times U$, where $U$ is the unit interval, then there are only two maximal chains in $\lambda$ while there are infinitely many maximal chains in $\rho$. It is apparent that the new representation is considerably more economical than the old.

\section{BIBLIOGRAPHY}

1. O. Ore, Galois connexions, Trans. Amer. Math. Soc. vol. 55 (1944) pp. 493-513.

2. G. Birkhoff, Lattice theory, rev. ed., Amer. Math. Soc. Colloquium Publications, vol. 25, 1948.

3. C. J. Everett, Closure operators and Galois theory in lattices, Trans. Amer. Math. Soc. vol. 55 (1944) pp. 514-525. 289.

4. G. Pickert, Bemerkungen über Galois-Verbindungen, Arch. Math. vol. 3 (1952) pp. 285-

5. P. Dubreil and R. Croisot, Propriêtés générales de la résiduation en liaison avec les correspondances de Galois, Collect. Math. vol. 7 (1954) pp. 193-203.

6. G. N. Raney, A subdirect-union representation for completely distributive complete lattices, Proc. Amer. Math. Soc. vol. 4 (1953) pp. 518-522.

7. J. Riguet, Relations binaires, fermetures, correspondances de Galois, Bull. Soc. Math. France vol. 76 (1948) pp. 114-155.

8. G. Aumann, Bemerkungen über Galois-Verbindungen, Bayer. Akad. Wiss. Math. Nat. Kl. S. B. (1955) pp. 281-284.

The Pennsylvania State University, University Park, Pennsylvania 